\title{
Poly(vinylalcohol)-borate hydrogels with improved features for the cleaning of cultural heritage surfaces
}

\author{
Chiara Riedo ${ }^{*}$, Fabrizio Caldera, Tommaso Poli and Oscar Chiantore
}

\begin{abstract}
:
Poly(vinylacohol) (PVA) is a versatile polymer capable of forming hydrogels useful in a wide range of applications. Hydrogels based on cross-linking of PVA with borax, and able to incorporate organic solvents, have been used in the field of cultural heritage as tool for cleaning the surfaces. Poly(ethylenoxide) (PEO) has been introduced in the formulations of PVA-borax, with the aim of increasing the gel compatibility with organic solvents and of improving the mechanical properties of the materials. The formulations were investigated with rheological characterization, solvent loss measurements and study of the free water through the determination of the Free Water Index. The PEO molecules do not participate in the formation of the network but have been found to be responsible for a decrement of the storage moduli, explained by a spacing between crosslinks and the partial disentanglement of the PVA chains. At the same time PEO produces in the gel an increase of the apparent relaxation time, thus stabilizing the dispersions and enhancing their ability to maintain the shape. Cleaning tests for removal of an acrylic coating on limestone have been also successfully performed.
\end{abstract}

Keywords: Poly(vinyl alcohol), Hydrogels, Polymer gels, Rheology, Cleaning in cultural heritage

\section{Background}

Poly(vinyl alcohol) (PVA) is an attractive polymer because of its many characteristics making it suitable for various applications, ranging from pharmaceutical and biomedical to industrial uses. Of particular interest is the PVA capability of forming a variety of hydrogel systems, which may be physically or chemically cross-linked, further expanding the possible applications of this versatile polymer. It is well known that polymers with hydroxyl groups form complexes with various ions such as borate [1-4] and vanadate [5]. PVA-ions complexation induces the formation of gel-like materials, capable of incorporating large amounts of water. The poly(vinyl alcohol) (PVA)-borate gel systems have received much attention because of their interesting chemical and physical properties and widespread industrial interests. The crosslinking mechanism of PVA with borate is known as "di-diol"

*Correspondence: chiara.riedo@unito.it; oscar.chiantore@unito.it Department of Chemistry and NIS Interdepartmental Center, University of Torino, via Pietro Giuria 7, 10125 Turin, Italy complexation, and involves two diol units and one borate ion. The kind of linkage which forms has been largely debated and in particular two models have been proposed. The first one suggests that the linkage between diols and borate ions has a mixed physical and chemical nature [6], while the second one implies only dynamic chemical cross-links between PVA chains and borate ions [7-9] and considers two types of interaction: monodiol complexation and crosslinking. After reaction the polymer chain behaves as a polyelectrolyte with electrostatic repulsions, resulting in an expansion of the network [10]. In semidilute solutions both intra and intermolecular cross-links may form, causing gelation of the PVA-borate complex [11].

PVA-borate hydrogels exhibit frequency-dependent mechanical behavior and finite relaxation time and consequently from the rheological point of view they are not considered real gels [12, 13], but instead viscoelastic dispersions with a dynamic network. The two frequencydependent moduli G' (storage modulus) and G" (loss modulus) are used to characterize the viscoelasticity

\section{Chemistry Central}


of the resulting material. PVA-borate hydrogels exhibit viscous behavior at a long time scale (low frequency), at which the network has sufficient time to rearrange (or disentangle) and elastic behavior at short time scale (high frequency), at which crosslinks cannot dissociate [4]. Several studies report that the increase of borate ions concentration contributes to the dispersion strength less than the polymer molecular weight and concentration $[14,15]$, which therefore appear to be the dominant factors contributing to the elastic behavior and stability of the dispersions.

The viscoelastic features of this kind of high viscosity polymeric dispersions (HVPDs) make them suitable, among other applications, for cleaning purposes in the field of cultural heritage. Cleaning is here intended as removal of generic dirt, products of degradations of original materials (e.g. aged varnish), coating applied in precedent restauration operations (e.g. protective materials or repainting) and all of those materials considered extraneous to the original work. Traditional cleaning of artistic surfaces involves the use of free solvents, applied with cotton-wool swab or small brushes. This system does not provide control over capillary flow through the surface. For painted surfaces this flow may causes swelling of pictorial layers and undesired removal of original materials. When used to remove layers of dirt or coating from particularly porous surfaces like stone or wood, free solvents may cause penetration of dissolved layers in the bulk of materials, making impossible further removal procedures. In addition, other limitation factors of solvent cleaning are the difficulty of removing layers in a controlled selective way, the unknown long-term effects of leaching and swelling of the paint layers, and the eventual toxicity of liquids [16]. In the 1980s Richard Wolbers introduced the use of the so-called solvent gels, which are hydrogels containing organic solvents. These preparations, based on poly(acrylic acid) as a gelator, minimize the negative effects related to the penetration of liquid, but some residues can remain on the surface after the cleaning process [17]. PVA/borax hydrogels, on the other hand, show mechanical features suitable for easy removal by peeling from surfaces, without need of strong mechanical or chemical actions, and have been therefore proposed for cleaning purposes [14, 18-20]. Water/ cosolvent mixtures can be embedded into these high viscosity polymeric dispersions, making them able to soften and swell water-insoluble materials, in a way similar to that proposed in a study concerning the solubilization of hydrophobic substances by hydrotropes in water [21].

Being PVA a very polar polymer, not fully compatible with the organic solvents of interest, modified formulations have been also proposed where PVAs with lower degree of hydrolysis are used [20]. PVA is in fact produced by polymerization of vinyl acetate followed by hydrolysis of the resulting poly(vinyl acetate). The hydrolysis does not generally go to completion resulting in polymers with remnant vinyl acetate units in the polymer chains. Different PVA grades are commercially available and the degree of hydrolysis, or the content of acetate, has an overall influence on their chemical properties, solubility and crystallization capability [22].

The presence of water miscible organic solvents in the PVA/borax gels has been shown to strongly modify the gel physical properties $[18,20]$, and when the application to cleaning of artistic surfaces is concerned, the main interest is to obtain controlled rheological properties and to expand the compatibility with organic solvents, starting from known formulations and modifying them by the addition of suitable additives. In the present study we have investigated the effect of adding poly(ethyleneoxide) (PEO) in various concentrations on the mechanical properties of PVA/borax gels and its influence on the capability of retaining organic solvents. PEO is a water-soluble and biocompatible thermoplastic polymer and the literature suggests that it causes an increment of the pores size in PVA/borax hydrogels $[23,24]$ with increasing, at the same time, the mechanical properties. For the above reasons $\mathrm{PEO}$ could be a suitable additive for improving the physical characteristics of PVA/borax hydrogels, and possible positive interactions with the miscible organic solvents used in conservation applications.

\section{Methods}

Poly(vinyl alcohol) (86-89\% hydrolyzed, Mw 100000, Fluka, Italy), poly(ethyleneoxide) (Mw. 35000, SigmaAldrich, Italy), sodium tetraborate decahydrate (SigmaAldrich, Italy), 2-propanol (Sigma-Aldrich, Italy) and acetone (Sigma-Aldrich, Italy) were used as received. Paraloid ${ }^{\circledR}$ B72 (pellets) is an ethyl methacrylate-methyl acrylate copolymer produced by Rohm and Haas and it was purchased from CTS, Italy. The limestone specimens were kindly supplied by Institute for the Conservation and Valorization of Cultural Heritage "Sezione Milano" of National Research Council (ICVBC-CNR, Italy).

Preliminary tests with PVAs having different molecular weights have shown that a molecular weight of about 100,000 is suitable to obtain an hydrogel with the desired mechanical properties in a polymer concentration not over $3-4 \%$ in weight and with a borax concentration not exceeding $1 \%$. Low polymer and crosslinker concentrations are required in order to have easy dissolution in water and in the water/cosolvent mixtures. On the other hand, for application on cultural heritage surfaces, optimum formulations are those with minimum amount of 
solid materials, in order to prevent contamination of the surfaces. For PEO the choice of 35,000 MW was made for having good solubility in the liquid phase.

The gels were prepared with different concentrations of PVA, borax and PEO, with deionized water only and with addition of cosolvents (see Table 1). The preparation procedure was the same for all formulations: the proper amounts of PVA and PEO were heated in deionized water at $70-80^{\circ} \mathrm{C}$ under magnetic stirring until complete dissolution. Sodium tetraborate decahydrate (borax) was solubilized in deionized water at $6 \%$ weight concentration. The borax solution was then added at room temperature and under vigorous stirring to the polymer solution, until the final salt concentration. With the addition of borax the gel forms instantaneously. When a formulation includes the presence of cosolvent, the latter is added after cooling of the polymer solution, to prevent evaporation. Formulations were left to stand 2 days before use, to allow expulsion of air bubbles.

Evaporation of liquid phase from gels was measured by placing few mg of sample on alumina pan and using the microbalance of a TGA instrument. The furnace of the TGA was closed and no flux of gas was used, to reproduce evaporation in normal atmospheric condition. Measurements were performed in triplicate at room temperature for $30 \mathrm{~min}$.

The water state in the gels was determined with a DSC Q200 (TA Instruments) equipment [25, 26]. Weighted amount of samples (between 5 and $10 \mathrm{mg}$ ) were loaded in sealed aluminum pans and cooled until $-60^{\circ} \mathrm{C}$. Heating rate was $5^{\circ} \mathrm{C} / \mathrm{min}$, up to $30^{\circ} \mathrm{C}$. Reference sample was an empty sealed aluminum pan. Endothermic peaks were integrated to obtain enthalpy changes of water melting. The gels tested contain $3 \%$ of PVA, $0.6 \%$ of borax, variable amounts of $\mathrm{PEO}$ and different liquid phases (water

\section{Table 1 Composition of the gel formulations}

\begin{tabular}{|c|c|c|c|}
\hline PVA \% & PEO \% & Borax \% & Cosolvent \% \\
\hline 3 & 0 & 0.6 & $\begin{array}{l}\text { No } \\
\text { 30\% 2-propanol } \\
\text { 30\% acetone }\end{array}$ \\
\hline 3 & 1 & 0.6 & $\begin{array}{l}\text { No } \\
\text { 30\% 2-propanol } \\
\text { 30\% acetone }\end{array}$ \\
\hline 3 & 2 & 0.6 & $\begin{array}{l}\text { No } \\
\text { 30\% 2-propanol } \\
\text { 30\% acetone }\end{array}$ \\
\hline 3 & 3 & 0.6 & $\begin{array}{l}\text { No } \\
\text { 30\% 2-propanol } \\
\text { 30\% acetone }\end{array}$ \\
\hline 2 & 0 & 0.4 & $\begin{array}{l}\text { No } \\
30 \% \text { acetone }\end{array}$ \\
\hline
\end{tabular}

or water/acetone mixture in the ratio 70/30). Data are reported as average of three repeated measurements for each type of formulation.

Oscillatory rheological measurements were carried out on a TA Instruments Discovery HR 1 equipped with $20 \mathrm{~mm}$ plate geometry and Peltier plate temperature control. Frequency sweep test were performed over the frequency range of $0.005-15 \mathrm{~Hz}$ and stress amplitude of $2 \%$. Value of stress amplitude were preliminary checked with amplitude sweep test, to ensure that measurements were performed within the linear viscoelastic region. Samples were equilibrated for $30 \mathrm{~min}$ prior the experiments to allow relaxation of material and temperature equilibration $\left(20^{\circ} \mathrm{C}\right)$. Three measurements were performed for each sample and the mean values are reported together with their standard deviations.

\section{Cleaning tests}

Cleaning tests were performed to compare the effectiveness of removal of gels with different formulations. In particular, gels prepared with $3 \%$ of PVA, $0,6 \%$ of borax and $30 \%$ of acetone, with and without PEO, were measured to check PEO effects on the gels performance.

The removal of the gels was tested on limestone specimens coated with Paraloid B72; the stone surfaces were particularly rough and therefore suitable to produce severe conditions for the peeling of gel. The high porosity of the limestone was also appropriate to test the efficacy of the gel in retaining the liquid phase. To evaluate the ability of the gels in removing the Paraloid without causing its penetration in the bulk of the specimens, capillary absorption measurements were performed on the raw stone, after Paraloid deposition and after treatment with the gels [27].

Specimens for cleaning test were prepared with limestone samples of $5 \times 5 \times 1 \mathrm{~cm}$. Paraloid B72 was dissolved in acetone at $2 \%$ weight concentration. Three layers of solution of Paraloid B72 were put on limestone samples, to ensure the formation of a solid film on the stone surface. For capillary absorption measurements stone samples were put in contact with water through a multiple layer of filter papers and the water absorbed was determined with gravimetric measurements carried out at definite time intervals. The absorption curves report results in terms of $\mathrm{mg} / \mathrm{cm}^{2}$ versus time $\left(\mathrm{s}^{1 / 2}\right)$.

For the cleaning tests an amount of gel was molded to cover the stone surface treated with the Paraloid B72. The gel was covered with a glass slide to prevent solvent evaporation and was removed after few minutes by the aid of tweezers. The stone specimen submitted to the gel action was left to dry at room temperature before measuring the water absorption. 


\section{Results and discussion}

The composition of the different gels investigated is reported in Table 1. All formulations prepared with 3\% of PVA showed optical transparence and mechanical properties suitable to be easily "peeled" after application (Figure 1a), whereas dispersions with $2 \%$ of PVA are too fluids to be handled (Figure 1b).

PVA and PEO are not completely miscible [28, 29], and to obtain a stable and transparent gel formulation the critical point is the perfect dissolution of the polymers in the common solvent. When dissolution is not complete the gels are opaque and undergo premature syneresis. Application and removal tests were done on microscope glass slides. Preliminary evaluations of the mechanical behavior of the gels have been made by simple visual observation of their plasticity and removal characteristics. Formulations with water alone and with water/2propanol demonstrate handling properties that do not vary with changing the $\mathrm{PEO}$ concentration in the range of $0-3 \%$. Instead, the addition of PEO makes easier the workability and application on the slide surface of the

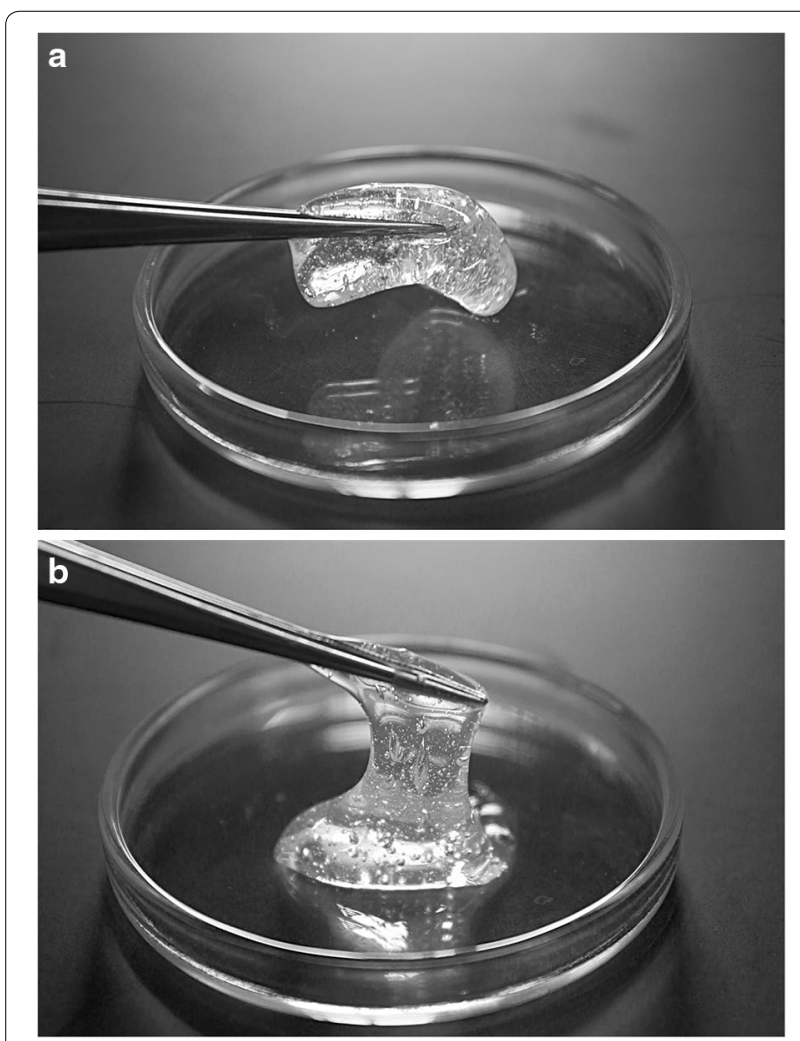

Figure 1 Comparison between mechanical behavior of hydrogel with $3 \%$ of PVA and $0.6 \%$ of borax (a) and hydrogel with $2 \%$ of PVA and $0.4 \%$ of borax $(\mathbf{b})$. formulations containing acetone. This is a positive effect, as the dispersions with water/acetone mixture are more rigid and less easily spreadable in thin layers than the same formulations containing water only. These observations have been corroborated by the rheological results, as described below.

\section{Liquid phase evaporation}

The ability of the formulation to retain the solvent during the cleaning process is an important requirement. Measurements of the liquid phase evaporation from the gels, opportunely layered on a glass slide to simulate the application on a flat surface, were done by gravimetry. The weight loss of the material is assumed to be due only to the evaporation of the liquid phase and was monitored up to $30 \mathrm{~min}$ of exposure.

As shown in Figure 2 in the formulations with water and water/2-propanol mixtures the retention of liquid phase is not affected by the PEO content. The high polarity of the liquid phase in these formulations is probably responsible of this behavior: because of the liquid phase good compatibility with PVA, the introduction of a less polar additive (PEO) does not interfere with the interactions controlling the liquid retention. A different situation appears to develop when the polarity of the cosolvent is lower, as it happens in the case of acetone. In Figure 2 it may be seen that the addition of PEO is advantageous and gels with this additive show better retention features than formulations with PVA only. The effect is not very strong and is not function of the PEO concentration as the best performance in terms of solvent retention is achieved with the PEO content of $1 \%$. An increment in solvent retention is important for a better control of the

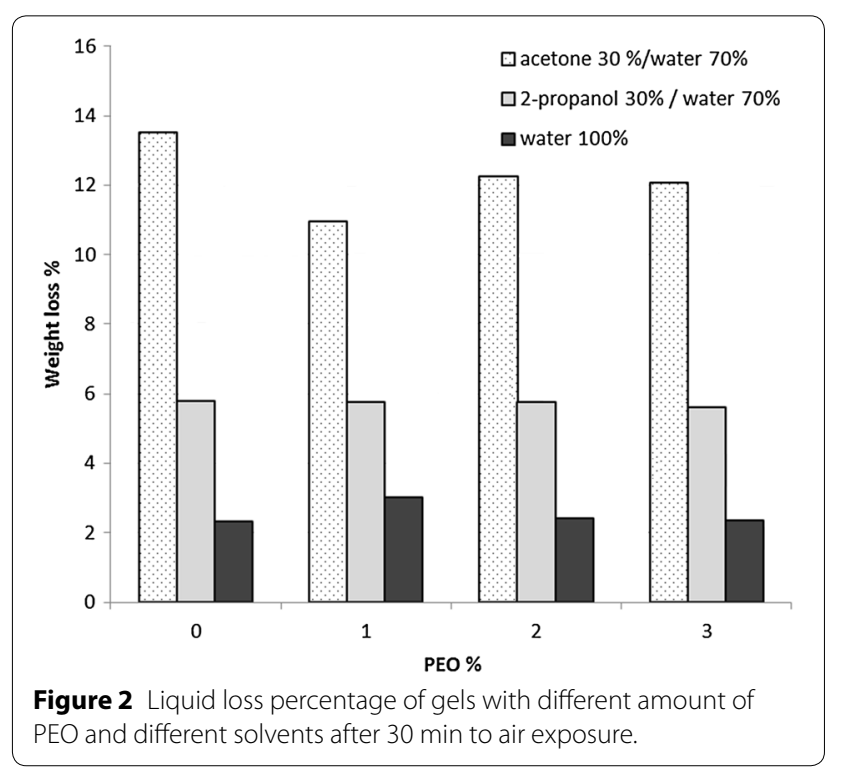


liquid composition which comes in contact with the surface to be cleaned.

\section{Characterization of the water state in the gel networks}

The water state in the HVPDs is expected to depend from the internal microstructure of the polymer network and the extent of the interactions which can develop with the gellant polymer molecules in particular. It is well established that in the hydrogel polymeric networks the water is present in three different states: free water, intermediate water and bound water [25]. Free and intermediate water are considered freezable, whereas bound water is considered nonfreezable and they may be differentiated based on the different freezing temperature and melting enthalpy. The state of water in polymeric dispersion may be determined through the use of the Free Water Index. The melting enthalpy of water is derived from DSC thermograms, by integration of the melting endothermic peak and is used to calculate the FWI from the following equation [18]:

$$
\mathrm{FWI}=\Delta \mathrm{H}_{\mathrm{m}} / \mathrm{W}_{\mathrm{f}} \Delta \mathrm{H}_{\mathrm{t}}
$$

where $\Delta \mathrm{H}_{\mathrm{m}}$ is the measured enthalpy of water melting, $\mathrm{W}_{\mathrm{f}}$ the water weight fraction in the gel and $\Delta \mathrm{H}_{\mathrm{t}}$ the theoretical value of the enthalpy of fusion of water at $0^{\circ} \mathrm{C}$. Low values of FWI indicate large amounts of bound structured water in the gel.

In Figure 3 the FWI for systems containing the same amount of PVA and two different compositions of liquid phase, water only and water/acetone, are plotted as a function of the PEO added concentration. The most evident difference is the drastic decrease of FWI in the formulations including the organic solvent, in strict agreement with results reported in the literature [14, $18,19,25]$. In the concentration range here investigated,

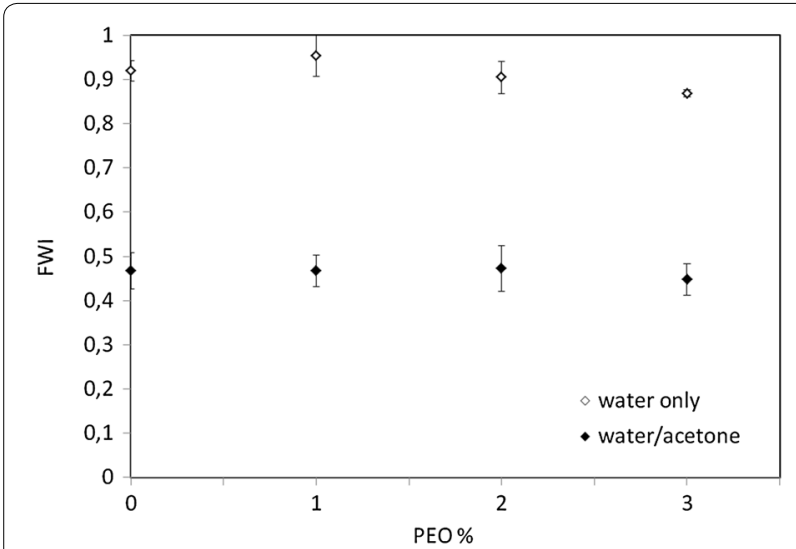

Figure 3 Free Water Index as a function of PEO content. Standard deviations are reported as error bars. the addition of PEO does not have much effect on FWI. Suggestions have been advanced about the decrease of the FWI induced by the presence of an organic content in the liquid, as being the result of a different (increased) structuring of the water in the system [14, 19]. Different types of dynamic polar interactions have been shown to develop between the molecules in mixtures of acetone and water [30] and it appears that as a result of these interactions in the gel environment more water becomes bound to the polymer strands. The addition of PEO, on the other hand, practically does not affect significantly the amount of bonded water.

\section{Rheological characterization}

The main purpose of the rheological measurements was to find the correlation between the mechanical properties and the addition of PEO in the formulations. The data obtained from the measurements also allow to make some considerations about the structural properties, which are closely related to the composition of the formulations in terms of polymers and organic solvent concentrations.

All acetone containing formulations, that are of major interest for the cleaning purposes, and some formulation with water only were submitted to oscillatory rheological measurements. As expected, a dependence of storage and loss modulus from frequency is observed (see mechanical spectra in Figures 4, 5). It is also evident that the mechanical curves cannot be fitted by a simple model with a unique relaxation time, which is not surprising in complex structured fluids like those considered in the present study where the relaxation mechanism is influenced by the interaction of different components.






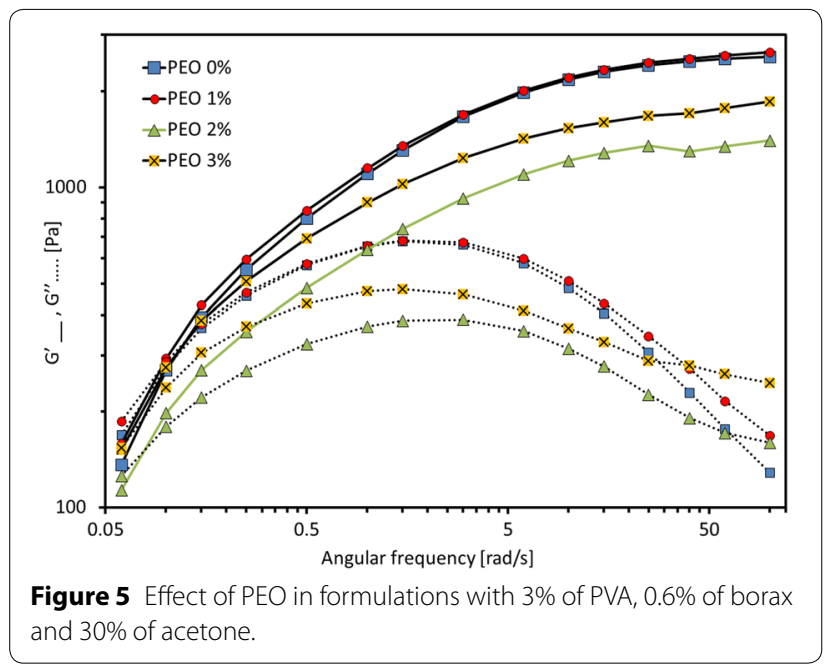

For such reason the terminal relaxation time could not be extracted from the oscillatory measurements and to characterize the mechanical spectra the crossover coordinates were therefore used.

The crossover coordinates (crossover frequency $\omega_{\mathrm{c}}$ and crossover modulus $G_{c}$ ) and the asintotic value of storage modulus $\left(\mathrm{G}_{0}^{\prime}\right)$, as described in the introduction, are useful to characterize the viscoelasticity of the HVPDs. From the crossover frequency $\left(\omega_{c}\right)$ it is possible to calculate an apparent relaxation time $\left(\tau_{\mathrm{c}}\right)$, related to the crosslink dynamics, with the following expression [31]:

$$
\tau_{c}=2 \pi / \omega_{c}
$$

The longer the gel relaxation time the more pronounced are the elastic features and the shape-stability.

In complex fluids the trend of storage modulus $\mathrm{G}^{\prime}$ versus frequency is asymptotic and $\mathrm{G}^{\prime}$ becomes independent from frequency in correspondence of the intrinsic elastic modulus $\mathrm{G}_{0}^{\prime}[32]$. $\mathrm{G}_{0}^{\prime}$ value is directly related to the gel peelability and the limit suggested in the literature for obtaining peeling is about $400 \mathrm{~Pa}$, at the typical perturbation frequencies induced during the gel removal in cleaning operations (around few hertz) [20]. Gels prepared with 2\% PVA concentration, with and without acetone, show $G_{0}^{\prime}$ values of 100 and 370 Pa respectively (see Table 2) and both are not peelable, in accordance to the above observations. At this concentration, the overlap between polymer chains is not sufficient to obtain an extensive interchain crosslinking, responsible of the elastic character of the material.

If from the macroscopic point of view $\mathrm{G}_{0}^{\prime}$ is directly related to the gels peelability, from the structural aspect it is connected to the entanglement/crosslinking density $\rho_{\mathrm{e}}$ according to the relation [33, 34]:

$$
\mathrm{G}_{0}^{\prime}=\rho_{\mathrm{e}} \mathrm{k}_{\mathrm{b}} \mathrm{T}
$$

where $k_{b}$ is the Boltzmann's constant and $T$ the temperature.

Raising the concentration of gellant polymer from 2 to $3 \%$ a drastic increment in $\mathrm{G}_{0}^{\prime}$ and in relaxation time is observed, as reported in Table 2. In the formulations with $3 \%$ of PVA, with $G_{0}^{\prime}$ values ranging from 1,000 to $3,000 \mathrm{~Pa}$, easily peelable materials are obtained and no macroscopic residues were observed after their removal from the glass slides. When cleaning operations of artistic surfaces are involved, it is also important to consider the ease of application and the thinning behavior of the gel, together with its ability to preserve the shape during the time necessary for the cleaning action. To understand this feature it is necessary to take into account the rheological behavior below the crossover point, in the region where formulations show their viscous behavior. If apparent relaxation times are long, the formulations display strong elastic behavior also in the range of low frequencies, therefore their applicability may be problematic. Most of the formulations containing acetone, in Table 2, have apparent relaxation time near or above $1 \mathrm{~min}$ and their structure is particularly stable, thus resulting in a difficult application to surfaces. The formulations without acetone have apparent relaxation time in the order of $10 \mathrm{~s}$ and their thinning is easily obtainable. However in

\begin{tabular}{|c|c|c|c|c|c|c|c|c|c|c|}
\hline PVA \% & PEO \% & Acetone \% & $\mathrm{G}_{\mathrm{c}}$ & RSD \% & $\omega_{c}(\mathrm{rad} / \mathrm{s})$ & RSD \% & $\tau_{c}(s)$ & RSD \% & $\mathrm{G}_{0}^{\prime}$ & RSD \% \\
\hline 3 & 0 & 30 & 295 & 21 & 0.11 & 26.02 & 58 & 23 & 3,033 & 4 \\
\hline 3 & 1 & 30 & 272 & 19 & 0.09 & 26.10 & 67 & 22 & 2,649 & 13 \\
\hline 3 & 2 & 30 & 150 & 43 & 0.08 & 16.97 & 82 & 16 & 1,401 & 10 \\
\hline 3 & 3 & 30 & 160 & 10 & 0.06 & 5.06 & 100 & 5 & 1,852 & 11 \\
\hline 3 & 1 & 0 & 283 & 13 & 0.49 & 2.4 & 13 & 2 & 1,135 & 11 \\
\hline 2 & 0 & 0 & 33 & 3 & 1.44 & 0.6 & 4 & 1 & 108 & 19 \\
\hline 2 & 0 & 30 & 74 & 5 & 0.36 & 7.6 & 18 & 5 & 369 & 6 \\
\hline
\end{tabular}

Table 2 Mechanical characteristics of the gels as obtained from the rheological measurements

Mean values and relative standard deviations of $G_{c}$ crossover modulus, $\omega_{c}$ crossover frequency, $\tau_{c}$ apparent relaxation time, $\mathrm{G}_{0}^{\prime}$ intrinsic elastic modulus). 
these cases the gels may have too strong tendency to flow and when the PVA concentration is lower than $3 \%$ the elastic moduli are not enough high to obtain peelability.

Acetone as cosolvent strengthens the gel network, and this effect may be evaluated from the increase of the storage and loss moduli and the relaxation times in comparison to the gels with only water. In Figure 4 the mechanical spectra of two formulations with different composition of liquid phase are shown and it is evident that dispersions containing acetone display much stronger elastic behavior than formulations with water only. This effect has been interpreted $[14,20]$ by means of ${ }^{11} \mathrm{~B}-\mathrm{NMR}$ measurements showing that in the presence of organic solvent the concentration of boron species bounded with the vinyl alcohol groups is enhanced. The environment created by the organic liquid is less attractive to the borate ions, forcing them to increase the complexation with PVA chains. The organic molecules in the liquid environment strongly affect the gel structures inducing a migration of borate ions towards PVA chains, with subsequent increase in the crossover modulus. The rheological results are in agreement with the FWI data obtained by DSC and reported in the previous section (Figure 3). The FWI decrement observed in the presence of acetone indicates an increment in the amount of structured water, directly related with the enhanced elastic character.

We may therefore conclude that the increased stiffness of the gels containing acetone, with constant PVA/borax ratio, is due to a larger amount of cross-links in comparison with the gels containing only water.

When PEO is added to the formulations the rheological properties of the gels undergo large changes, as shown in Figure 5 by the mechanical spectra of the gels containing different amounts of the polymeric additive. The relevant data from the mechanical spectra of Figure 5 are listed in Table 2, and a progressive decrement in $G_{0}^{\prime}$ is observed in these gels when the PEO concentration increases up to $2 \%$ (Figure 6a). The concentrations of cosolvent and of borax, considered the major responsible of the crosslinking formation, are unchanged in these formulations and, on the other hand, it was checked that PEO alone is not able to react with borate ions to form a network. We can therefore exclude that PEO can compete with the vinyl alcohol units for reacting with borate ions. From literature studies it is known that PEO acts as a porogen agent in PVA/borax membranes [23] and in PVA gels obtained with freezing/thawing method [24]. In the latter work is reported that addition of PEO in a PVA/water system causes the decrease of PVA degree of crystallinity resulting in the increase of network mesh size and decrease of the storage modulus. Our results are in agreement with the above findings suggesting that

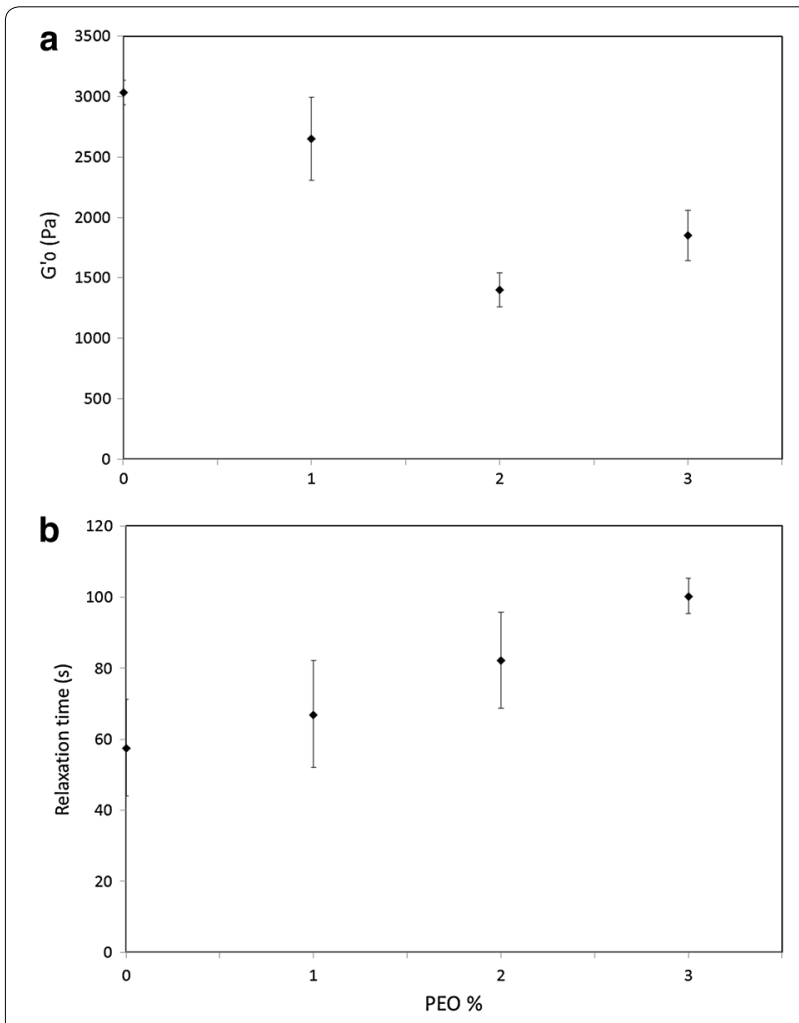

Figure $\mathbf{6} \mathrm{G}_{0}^{\prime}(\mathbf{a})$ and relaxation time $(\mathbf{b})$ as a function of PEO content. Standard deviations are reported as error bars.

dissolution of PEO molecules in the PVA hydrogels up to a given concentration produces an increase of the network mesh size through relaxation and partial disentanglement of the PVA chains, with a substantial change of the three-dimensional structure and an effective reduction of the total crosslink concentration considered as the sum of borate ions/hydroxyls complexation and polymer chain entanglements. This process may be considered the responsible for the observed reduction of the gels intrinsic elastic moduli.

The softening effect induced by the presence of the PEO molecules is at the same time accompanied by an almost linear increase of the gel relaxation times which are calculated from the mechanical spectra and plotted in Figure 6b. This behavior may appear contradictory, but it is necessary to consider that the fluid composition of the gel is now changing with the PEO addition, and the relaxation mechanisms are affected not only by the crosslink concentration but also by the surrounding fluid viscosity. In order to clarify the effect of PEO in the PVA/borax system it may be useful to compare the mechanical spectra not only from the quantitative point of view by considering the crosslinking parameters, but also the overall appearance of the curves. One method for comparing mechanical spectra is to normalize the 
spectra [35] but a simple way is to look at the $\tan (\delta)$ values, obtained from the ratio $\mathrm{G}^{\prime \prime} / \mathrm{G}^{\prime}$. Tan $(\delta)$ describes the capability of the materials to dampen the applied strain (or stress), that is the equilibrium result between conservation $\left(G^{\prime}\right)$ and dissipation $\left(G^{\prime \prime}\right)$ of the energy. When $\tan (\delta)$ is high $\left(G^{\prime \prime}>G^{\prime}\right)$ a material has stronger viscous character, on the contrary when $\tan (\delta)$ is low $\left(G^{\prime}>G^{\prime \prime}\right)$ in a materials the elastic behavior is dominant. The analysis of $\tan (\delta)$ values as a function of frequency can therefore give the same information obtainable from the comparison of $\mathrm{G}^{\prime}$ and $\mathrm{G}^{\prime \prime}$ curves.

If the $\tan (\delta)$ curves overlap it means that the relaxation mechanism of the different formulations is almost the same, even if the time scale is different. $\operatorname{Tan}(\delta)$ as a function of frequency for the formulations with different amounts of PEO are reported in Figure 7 . The dotted line in the figure represents the value $\tan (\delta)=1$, occurring at the crossover frequency. The data show that no complete overlap of the $\tan (\delta)$ curves is achieved, indicating that the relaxation mechanism of the different formulations changes for the presence of PEO. In particular it may be seen in the Figure that in the low frequency region $\tan (\delta)$ decreases with increasing PEO concentration. This means that PEO enhances the ability of the material to dampen the applied stress, causing an augmentation in the time relaxation. This may be attributed to the simultaneous presence of sol (PEO solution) and gel (PVA/ borate): the sol relaxes more quickly than the gel network but at the same time the presence of PEO enhances the viscosity of the fluid fraction in the formulation, slowing down the gel relaxation. On the contrary, in the high frequency region associated to the elastic character, the $\tan (\delta)$ becomes an increasing function with PEO concentration. Therefore PEO is responsible of a decrement in the elastic character, as also indicated by the decrement of the intrinsic elastic modulus $\mathrm{G}_{0}^{\prime}$.

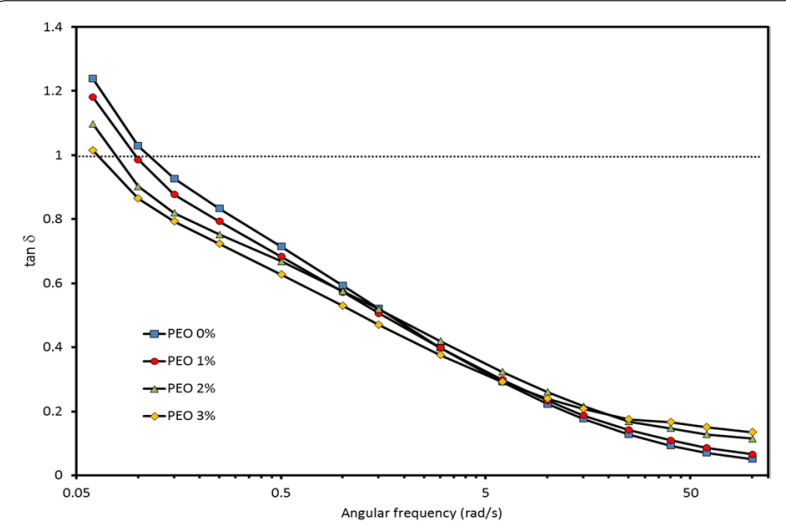

Figure $7 \operatorname{Tan}(\delta)$ as a function of frequency for formulation with $3 \%$ of PVA, $0.6 \%$ of borax, $30 \%$ of acetone and variable amount of PEO.
The rheological characteristics which have been here determined indicate that in the formulations containing both the organic solvent and PEO it is possible to control at the same time the stiffness of the gel, which has relevance for the easiness of application, and the response to external solicitation, which affects the shape stability and the removability of the gels.

\section{Cleaning tests}

Cleaning tests were performed to check the ability of the different gel formulations to remove Paraloid ${ }^{\circledR}$ B72 from limestone specimens. Limestone was chosen as a substrate being particularly porous and suitable for testing the "peelability" of the gel and the ability of the gel to retain the liquid phase. The two tested formulations (with and without PEO) show adequate mechanical properties that allow easy removal of the material after cleaning application, as it is shown in Figure 8. It is possible to see that, notwithstanding the roughness and porosity of the surface, the removal of the gel is complete and no evidence of residuals is visible. After use the gel keeps its mechanical properties and is still perfectly peelable.

The gel loses water (and solvent) during cleaning, as shown by the measurements in Figure 2, but during typical application times $(2-5 \mathrm{~min})$ the liquid evaporation is not sufficient to generate dramatic changes in the rheological properties. Also, the glass slide used to cover the gel during the cleaning tests further prevents liquid phase evaporation.

For such tests a gel with $1 \%$ of PEO was preferred on the basis of the evaporation measurements which showed for this formulation the highest capability to retain liquid phase.

Different times of application were checked, and the best results have been obtained with application of $4 \mathrm{~min}$.



Figure 8 Example of gel removal from a limestone specimen. 
The curves in Figure 9a, b report the capillary absorption of the raw stone, of the stone after the Paraloid B72 treatment and after the gels application. In order to verify the cleaning efficiency the measurements have been repeated on several stone specimens and in the Figure the experimental curves relative to a single experiment are shown for illustration. When a gel without PEO is applied (Figure 9a) the absorption curve is nearly superimposable to the absorption curve of the coated surfaces prior to the gel treatment. This means that the gel without PEO is not particularly efficient in the removal of the Paraloid B72. In the first part of the curve the rate of absorption is faster than in treated stone. Our hypothesis is that the acetone at the gel-stone interface easily solubilizes the Paraloid film transporting the solubilized material in the stone porosities. The Paraloid migrates from the surface to the bulk of the stone, allowing the water to be absorbed. A different situation is achieved when cleaning test are performed with formulation containing $1 \%$ of PEO. As observed in the liquid phase evaporation measurements, PEO can improve to a certain extent
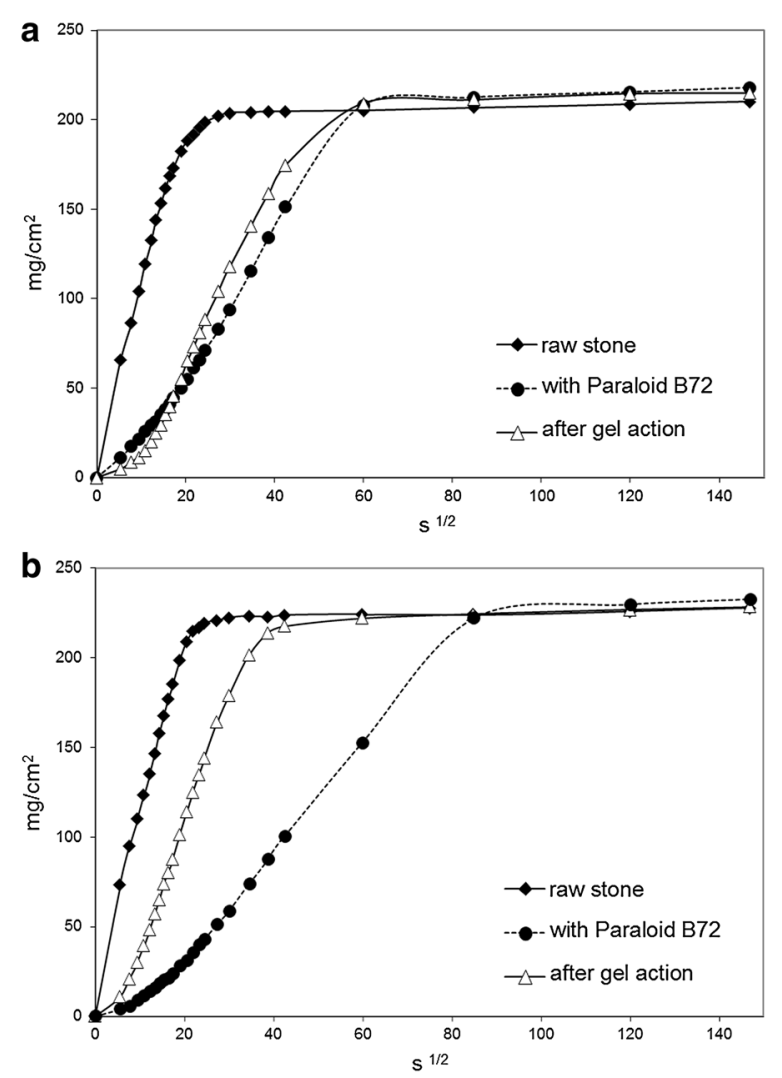

Figure 9 Water absorption curves of raw stone (diamonds), stone treated with Paraloid B72 (circles) and after cleaning test (triangles). Gels tested are composed by $3 \%$ of PVA, $0.6 \%$ of borax, $30 \%$ of acetone and $0 \%$ PEO (a) or 1\% PEO (b). the capability of the gel to retain the organic solvent. The liquid capillary absorption curves from the stone treated with Paraloid and from the same specimen after application of gel with PEO (Figure $9 \mathrm{~b}$ ) proves that PEO is really able to retain acetone in an extent useful to prevent the fast penetration trough the stone surface. In fact the curve, even if not coincident with the absorption curve of the raw stone, show that Paraloid was partially removed by the gel action: initial absorption rate is slower than in the raw stone, but faster than in the Paraloid coated surface. Moreover, after the initial absorption step, the absorption rate in the gel treated specimen is very similar to that in the raw stone, proving that the acrylic protective was indeed removed and not transported in the bulk porosity from acetone. Acetone is a good solvent for the Paraloid resin which was employed here and we may therefore assume that the removal of the protective film occurs by solubilization of the polymer and its incorporation into the gel matrix.

\section{Conclusions}

The influence of organic solvents on PVA/borate system was confirmed by rheological and DSC data, showing that they are able to strengthen the elastic behavior of the dispersions by increasing the crosslink density. When surface cleaning of art works is concerned this feature is positive for the gel removal, but not for its application because the increased stiffness can make difficult the thinning of the material. On the other hand, attempts to soften the formulation by decreasing the PVA content were not successful because with $2 \%$ only of gellant polymer the dispersions were too fluid to obtain peelability. Further studies to obtain correct equilibrium in the viscoelastic properties in presence of cosolvent are in progress and will be reported.

The addition of PEO to the dynamic network of PVA/ borate dispersion modifies the viscoelastic behavior of the gels and the liquid phase retention. The acetone retention, in particular, was improved with the presence of a limited amount of PEO in the liquid phase; the effect is not very strong, but the result is interesting as acetone is a solvent employed in many conservation treatments. Removal tests of a protective coating of Paraloid B72 from a limestone specimens was successful and confirm the capability of formulations with PEO to better retain the organic cosolvent. For this particular application the inhibition of solvent penetration is a crucial point because solvent absorption may cause the transport of solubilized polymer coating in the bulk of the stone, making irreversible any further attempt to remove the unwanted material. The cleaning tests show the absence of macroscopic visible residues on the stone specimens, but a crucial point for the gels used as cleaning systems 


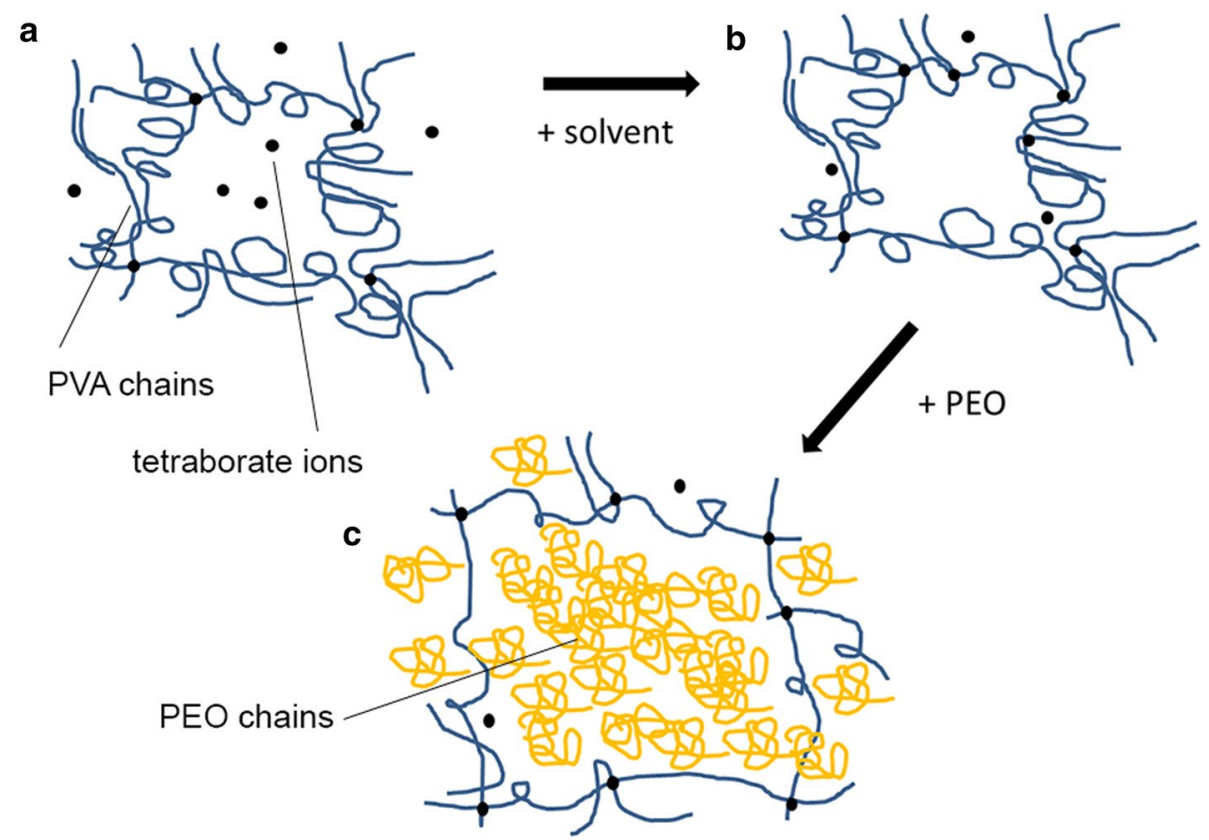

Figure 10 Proposed structure for HVPDs here discussed: a PVA and borate ions (dark circles); b effect of organic solvent; c effect of PEO. See text for explanation.

is whether micro-residues are retained on the substrates or not. A study about the presence of micro-residues is still in progress, with particular attention to the possible penetration into the bulk sample of the PEO, that is not chemically linked to the gel network.

The decrement of the storage moduli observed in the rheological characterization is explained by the spacing between crosslinking points and the partial disentanglement of PVA chains induced by the presence of PEO. At the same time the PEO molecules produce an increase of the apparent gel relaxation time, stabilizing the dispersions and enhancing their ability to maintain the shape. From the data here presented an hypothesis on the general structure of the HVPDs may be deduced, and is represented in Figure 10. As organic solvent and PEO are simultaneously added to the formulation it is presumed that the final structure is achieved in a single step but the effect of solvent and of PEO are depicted separately. In (a) only PVA and borate ions (dark circles) are present. In this situation an equilibrium between uncomplexed/ complexed borate ions is achieved. In (b) the presence of organic solvent induces a major amount of borate ions to be complexed, giving an increment in crosslink density and the consequent observed enhancement of intrinsic elastic modulus. In (c) the presence of PEO gives a partial disentanglement of the PVA chains with enlargement of the network size and no substantial modification in the borate ions crosslink density.
It is evident that when different liquids and different types of polymer molecules are introduced in the gel formulations, multiple interactions may develop among the components whose effects are not easy to predict. The gel structures, the related mechanical properties and the liquid phase retention can be largely influenced. The promising results obtained with the cleaning test encourage to carry on systematic structural and mechanical characterizations for tuning the formulations according the desired application. Applications on other materials usually present on artistic surfaces, such as natural or synthetic varnishes, and on aged and unaged specimens or on real manufacts, are in progress and will be soon documented.

\section{Authors' contributions}

All the authors contributed in the research activities. OC and CR were in charge of the planning and coordination of the activities. CR and FC prepared the formulations, and with TP performed the cleaning test. CR was responsible of data acquisition and analysis. The manuscript preparation and revision was done by $O C$ and CR. All authors read and approved the final manuscript.

\section{Acknowledgements}

Work accomplished with financial support from the National Research Project "Sustainability in cultural heritage: from diagnosis to the development of innovative systems for consolidation, cleaning and protection" (PRIN 2010-2011, MIUR, Italy).

\section{Compliance with ethical guidelines}

\section{Competing interest}

The authors declare that they have no competing interests. 
Received: 7 January 2015 Accepted: 2 July 2015

Published online: 03 August 2015

\section{References}

1. Ochiai H, Kurita Y, Murakami I (1984) Viscosity behavior of the polyelectrolyte poly(vinyl alcohol) having some intrachain crosslinks. Die Makromolekulare Chemie 185:167-172

2. Sinton SW (1987) Complexation chemistry of sodium borate with poly(vinyl alcohol) and small diols: a boron-11 NMR study. Macromolecules 20:2430-2441

3. Piest M, Zhang X, Trinidad J, Engbersen JFJ (2011) pH-responsive, dynamically restructuring hydrogels formed by reversible crosslinking of PVA with phenylboronic acid functionalised PPO-PEO-PPO spacers (Jeffamines). Soft Matter 7:11111-11118

4. Lin HL, Liu WH, Shen KS, LeonYu T, Cheng CH (2003) Weak gel behaviour of poly(vinyl alcohol)-borax aqueous solutions. J Polym Res 10:171-179

5. Shibayama M, Adachi M, Ikkai F, Kurokawa H, Sakurai S, Nomura S (1993) Gelation of poly(vinyl alcohol)-vanadate aqueous solutions. Macromolecules 26:623-627

6. Shibayama M, Hiroyuki Y, Kurokawa H, Fujiwara H, Nomura S (1998) Sol-gel transition of poly(vinyl alcohol)-borate complex. Polymer 29:2066-2071

7. Cheng ATY, Rodriguez F (1981) Mechanical properties of borate crosslinked poly(vinyl alcohol) gels. J Appl Polym Sci 26:3895-3908

8. Davis HB, Mott CJB (1980) Interaction of boric acid and borates with carbohydrates and related substances. J Chem Soc Faraday Trans 1(76):1991-2002

9. Pezron E, Leibler L, Lafuma F (1989) Complex formation in polymer-ion solutions. 2. Polyelectrolyte effects. Macromolecules 22:2656-2662

10. Lin HL, Liu YF, Yu TL, Liu WH, Rwei SP (2005) Light scattering and viscoelasticity study of poly(vinyl alcohol)-borax aqueous solutions and gels. Polymer 46:5541-5549

11. Chen CY, Yu TL (1997) Dynamic light scattering of poly(vinyl alcohol)borax aqueous solution near overlap concentration. Polymer 38:2019-2025

12. Burchard W, Ross-Murphy SB (1990) Introduction: Physical Gels from Synthetic and Biological Macromolecules. In: Physical Networks: polymer and Gels. Elsevier Applied Science, London

13. Almdal K, Dyre J, Hvidt S, Kramer O (1993) Towards a phenomenological definition of the term 'Gel' polymer. Polym Gels Netw 1:5-17

14. Angelova LV, Terech P, Natali I, Dei L, Carretti E, Weiss RG (2011) Cosolvent gel-like materials from partially hydrolyzed poly(vinyl acetate)s and borax. Langmuir 27:11671-11682

15. Kanaya T, Takahashi N, Nishida K, Seto H, Nagao M, Takeba Y (2006) Dymanic and static fluctuations in polymer gels studied by neutron spinecho. Phys B 385-386:676-681

16. Dodge V (ed) (2004) Solvent gels for the cleaning of works of art: the residue question. Getty Publications, Los Angeles

17. Burnstock A, Kieslich T (1996) A study of the clearance of solvent gels used for varnish removal from paintings. In: ICOM Committee for Conservation, 11th Triennial Meeting in Edinburgh, James \& James, London, pp 253-262
18. Carretti E, Grassi S, Cossalter M, Natali I, Caminati G, Weiss RG et al (2009) Poly(vinyl alcohol)-borate hydro/cosolvent gels: viscoelastic properties, solubilizing power, and application to art conservation. Langmuir 25:8656-8662

19. Carretti E, Natali I, Matarrese C, Bracco P, Weiss RG, Baglioni P et al (2010) A new family of high viscosity polymeric dispersions for cleaning easel paintings. J Cult Herit 11:373-380

20. Natali I, Carretti E, Angelova L, Baglioni P, Weiss RG, Dei L (2011) Structural and mechanical properties of "peelable" organoaqueous dispersions with partially hydrolyzed poly(vinyl acetate)-borate networks: applications to cleaning painted surfaces. Langmuir 27:13226-13235

21. Bauduin P, Renoncourt A, Kopf A, Touraud D, Kunz W (2005) Unified concept of solubilization in water by hydrotropes and cosolvents. Langmuir 21:6769-6775

22. Hassan CM, Peppas NA (2000) Structure and application of poly(vinyl alcohol) hydrogels produced by conventional crosslinking or by freezing/ thawing methods. Adv in Polym Sci 153:37-65

23. Zhang Y, Ye L (2011) Improvement of permeability of poly(vinyl alcohol) hydrogel by using poly(ethylene glycol) as porogen. Polym Plast Technol Eng 50:776-782

24. Lian Z, Ye L (2012) Effect of PEO on the network structure of pva hydrogels prepared by freezing/thawing method. J Appl Polym Sci 128:3325-3329

25. Lin H, Dan W, Dan N (2012) The water state in crosslinked poly(vinyl alcohol)-collagen hydrogel and its swelling behavior. J Appl Polym Sci 123:2753-2761

26. Ruiz J, Mantecon A, Cadiz V (2003) States of water in poly(vinyl alcohol) derivative hydrogel. J Polym Sci B 41:1462-1467

27. Norma UNI EN 15801—Cultural Heritage-Natural and artificial stonesDetermination of water absorption by capillarity (2010)

28. Mishra R, Rao K (1999) On the formation of poly(ethyleneoxide)/ poly(vinylalcohol) blends. J Eur Polym 35:1883-1894

29. Lai WC, Liau WB (2004) Study of the miscibility and crystallization behavior of poly(ethylene oxide)/poly(vinyl alcohol) blends. J Appl Polym Sci 92:1562-1568

30. Idrissi A, Longelin S, Sokolic F (2001) Study of aqueous acetone solution at various concentrations: low-frequency Raman and molecular dynamics simulation. J Phys Chem B 105:6004-6009

31. Piculell L, Egermayer M, Sjostrom J (2003) Rheology of mixed solutions of an associating polymer with a surfactant. Why are different surfactants different? Langmuir 19:3643-3649

32. Winter HH, Mours M (1997) Rheology of polymers near liquid-solid Transitions. Adv Polym Sci 134:165-234

33. Schubert BA, Kaler EW, Wagner NJ (2003) The microstructure and rheology of mixed cationic/anionic wormlike micelle. Langmuir 19:4079-4089

34. Gottlieb M, Macosko CW, Benjamin GS (1981) Equilibrium modulus of model poly(dimethylsiloxane) networks. Macromolecules 14:1039-1046

35. Carretti E, Matarrese C, Fratini E, Baglioni P, Dei L (2014) Physicochemical characterization of partially hydrolyzed poly(vinyl acetate)-borate aqueous dispersions. Soft Matter 10:4443-4449

\section{Publish with ChemistryCentral and every scientist can read your work free of charge \\ "Open access provides opportunities to our colleagues in other parts of the globe, by allowing anyone to view the content free of charge."}

W. Jeffery Hurst, The Hershey Company.

- available free of charge to the entire scientific community

- peer reviewed and published immediately upon acceptance

- cited in PubMed and archived on PubMed Central

- yours - you keep the copyright

Submit your manuscript here:

http://www.chemistrycentral.com/manuscript/ 